\title{
Attitudes of medical students and trainees about a career in Cardiothoracic surgery
}

Authors: Ariana Axiaq ${ }^{1 *}$, Raneesha Pillay ${ }^{2}$, Manasi Mahesh Shirke ${ }^{1}$, Sara Zaidi ${ }^{3}$, Amer Harky MRCS, $\mathrm{MSc}^{4,5,6,7}$

${ }^{1}$ School of Medicine, Queen's University Belfast, Belfast, UK

${ }^{2}$ Barts and The London, School of Medicine and Dentistry, London, UK

${ }^{3}$ King's College London, London, UK

${ }^{4}$ Liverpool Centre for Cardiovascular Science, University of Liverpool, Liverpool, UK

${ }^{5}$ Department of Cardiothoracic Surgery, Liverpool Heart and Chest Hospital, Liverpool, UK

${ }^{6}$ Liverpool Centre for Cardiovascular Science, University of Liverpool and Liverpool Heart and Chest Hospital, Liverpool, UK

${ }^{7}$ Department of Cardiac Surgery, Alder Hey Children Hospital, Liverpool, UK

DOI: https://doi.org/10.26800/LV-142-supp5-58

\section{Introduction:}

Cardiothoracic (CT) surgery is a dynamic and demanding speciality, which is popular amongst medical students thus, posing as a favourable career choice for many. However, a significant proportion of medical students prefer to choose other specialities instead, for various reasons.

\section{Objectives:}

The aim of this study is to identify factors affecting the uptake of cardiothoracic surgery as a career by medical students, junior doctors and trainees globally.

\section{Method:}

A comprehensive literature review was conducted using PubMed, EMBASE, SCOPUS and CINAHL using specific keywords including "cardiothoracic surgery" AND "medical student" AND "career". Inclusion and exclusion criteria were also developed to ensure only relevant studies were used for the paper. Information on the perspectives, knowledge, and beliefs on cardiothoracic surgery amongst medical students and trainee doctors worldwide was collected. Exposure to the speciality was also assessed.

\section{Results:}

Most data were sourced from the UK and US-based studies with only a minority of literature from other parts of the world. Uptake of cardiothoracic surgery amongst medical students, junior doctors and trainees are generally low, on a global level. Deterring factors identified from this review included work-life balance, professional satisfaction, lifestyle, and family planning, the latter being especially important for female medical students. Exposure to the speciality was generally low throughout medical school, better exposure was linked to higher interest in cardiothoracic surgery.

\section{Conclusion:}

Although job posts are still being filled, the increasing numbers of medical students losing interest in a career in cardiothoracic surgery needs to be addressed. Areas of future research into this area would be to re-evaluate medical school curricula and opportunities to engage more in the field whilst at medical school and beyond.

Keywords: cardiothoracic surgery, career, interest, medical students, trainees 